\title{
The Clever Use of Pressure in Column Liquid Chromatography
}

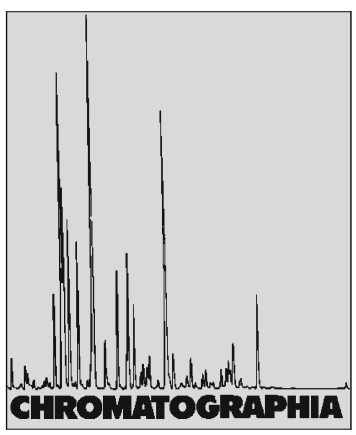

2010, 72, 603-609

\author{
Veronika R. Meyer ${ }^{\bowtie}$ \\ EMPA St. Gallen, Swiss Federal Laboratories for Materials Science and Technology, Lerchenfeldstrasse 5, 9014 St. Gallen, Switzerland; \\ E-Mail: veronika.meyer@empa.ch
}

\begin{abstract}
The pressure limits of a liquid chromatographic instrument can be maxed out by running the separation at the highest possible flow rate. This approach reduces analysis time but it does not save solvent and the separation will be poorer due to the properties of the van Deemter curve. Thus, it is better to use a shorter column with smaller particle size because both analysis time and solvent consumption will decrease while the resolution will remain constant. This paper shows how to utilize the pressure which is offered by a certain LC instrument in a clever way. It explains the algebraic background and illustrates the validity of the approach with two analytical problems, namely the separation of seven doping agents and of six drugs.
\end{abstract}

\section{Keywords}

Column liquid chromatography

Fast liquid chromatography

Pressure optimization

Isocratic mode

Method transfer

\section{Introduction}

Decades ago the term HPLC was the abbreviation of high pressure liquid chromatography. This alone makes clear that pressure is an essential issue in column chromatography with liquid mobile phases: high separation power is only possible with small stationary phase particles (due to the rather slow diffusion coefficients of the analytes in liquids) and long columns. This combination results in working pressures that usually exceed 100 bar. High-pressure pumps and other pressure-resistant instrumentation appeared on the market and were improved constantly, eventually mitigating the pressure problem for the time being.

However, the need for the separation of samples of increasing complexity, e.g. in proteomics or in environmental analysis, has recently led to the commercial introduction of pumps and systems which can be used up to 1,200 bar $[1,2]$. For these types of high-pressure applications (and also the ones run at 600 bar or the like) the term UHPLC was created, ultra high pressure liquid chromatography (sometimes also referred to as UPLC). Many users of LC and UHPLC systems are tempted to accelerate their separations in a thoughtless way simply by increasing the flow rate in order to decrease the analysis time, without taking into consideration the detrimental consequences this has on chromatographic resolution.

The way that diffusion coefficients, packing geometry and eluent flow interconnect is quite complicated and was first explained by van Deemter [3] and later adapted for LC by Knox [4]. These works show that there is an optimum flow rate for chromatographic mobile phases in any given set of parameters. A flow rate that is too slow results in excessive band broadening as well as long analysis time, making it highly unattractive. High flow 
rates lead to poorer separations as well although the effect is much less pronounced under such conditions. In between these extremes, there exists an optimum flow rate which yields the lowest possible theoretical plate height (or the highest number of theoretical plates) for a given chromatographic system and analyte. In many cases this value lies in the vicinity of:

$v=\frac{d_{\mathrm{p}} \cdot u}{D_{\mathrm{m}}} \approx 3$

where $v$ is the so-called reduced velocity of the mobile phase, $d_{\mathrm{p}}$ is the particle diameter of the column packing, $u$ is the linear velocity of the mobile phase (e.g. in $\mathrm{mm} / \mathrm{s}$ ), and $D_{\mathrm{m}}$ is the diffusion coefficient of the analyte in the mobile phase. If $d_{\mathrm{p}}$ and $D_{\mathrm{m}}$ are known (the latter can be estimated after Wilke and Chang [5]), it is then straightforward to calculate the optimum linear velocity $u_{\mathrm{opt}}$ with $\mathrm{Eq} 1$ or the corresponding volume flow rate $F$ (e.g. in $\mathrm{mL} \mathrm{min}^{-1}$ ) with:

$F=\frac{u \cdot d_{c}^{2} \cdot \pi \cdot \varepsilon}{4}$

where $d_{\mathrm{c}}$ is the column inner diameter and $\varepsilon$ is the total porosity of the column packing.

Of course, the van Deemter optimum can also be found experimentally by varying the flow rate and determining the peak widths or the number of theoretical plates as was done in this paper.

As early as 1974, Guiochon et al. [6-8] demonstrated that columns run at their van Deemter optimum make the best use of the available pressure. "It can be seen that there is always a minimum pressure for any given analysis and this optimum corresponds to the optimum velocity" [7]. It is obvious, therefore, that it would always be best to run any chromatographic column at its van Deemter optimum, resulting in the best performance and the lowest pressure which makes sense (as already mentioned, an ever lower pressure gives decreased performance and longer analysis times). Halász and Görlitz visualized the opportunities presented by columns run at their optimum by publishing two nomograms [9] (also shown in [10]). They allow to determine possible combinations of column length, particle diameter, pressure, breakthrough time, and theoretical plate number for both low-viscosity (i.e. normal-phase) and high-viscosity systems (i.e. reversedphase), respectively. With the nomograms it is, e.g. easily possible to determine the optimum conditions with regard to column length and particle size if the pressure is to be changed while retaining the same value for the number of theoretical plates.

Nevertheless, most analyses are run well above the optimum because the analysts want fast separations. The loss in performance is usually low, although it is a measurable difference. This paper shows that such a simple approach leads to analysis times that are too long and to a level of solvent consumption that is too high compared to the clever approach. The outlined proposal works for all kinds of liquid chromatographic separations, being they performed at optimal speed or at another position in the van Deemter curve.

\section{Theoretical Background of the Clever Pressure Approach}

We will assume that a certain separation is performed so far with a column of length $L_{1}$, filled with a particulate packing of diameter $d_{\mathrm{p} 1}$, operated at a linear velocity $u_{1}$ (being this near the optimum or far away) by applying a pressure $\Delta p_{1}$. Under these conditions, the column yields a certain number of theoretical plates $N_{1}$, enough to solve the separation problem, or a certain height of a theoretical plate $H_{1}$. We will now look at the effects of increasing the pressure by a factor of $n$, either thanks to a new UHPLC pump or because the old pump is not yet running at its limit: $\Delta p_{2}=n \bullet \Delta p_{1}$.

If the same column is used at $n$-fold pressure the separation system moves along the right side of the van Deemter curve. The temporal width of the peaks decreases, therefore it may be necessary to adapt the integrator settings (sampling rate, time constant, etc.). The spatial width of the peaks (or their volume) will increase if the original separation was running at or above the van
Deemter optimum, leading to broader peaks and poorer resolution. (The opposite case is an original separation running at too low a velocity with regard to the optimum, conditions which should be avoided. But now the peaks may become narrower with increasing flow rate, depending on the position of the new velocity with regard to the van Deemter curve.) The retention volume (i.e. the solvent consumption) will be identical, but the analysis time will decrease $n$-fold. This is represented by the "trivial change" arrow shown in Fig. 1.

An alternative to this approach is to use a column with a finer packing $d_{\mathrm{p} 2}$. If it is run at the same reduced velocity, the linear velocity $u_{2}$ must be higher:

$u_{2}=\frac{d_{\mathrm{p} 1} \cdot u_{1}}{d_{\mathrm{p} 2}}$

If the columns have the same inner diameter, the relationship between the volume flow rates $F$ will be analogous at identical $v$ :

$F_{2}=\frac{d_{\mathrm{p} 1} \cdot F_{1}}{d_{\mathrm{p} 2}}$

The new column will not have the same length as the old one in order to keep the number of theoretical plates constant. $N$ is proportional to the length of a column and to the inverse of the particle diameter:

$N \propto \frac{L}{d_{\mathrm{p}}}$

as long as both columns have the same packing quality, resulting in a proportionally identical van Deemter curve, and are used at the same reduced velocity. Therefore, the new column with identical plate number will be shorter:

$L_{2}=\frac{L_{1} \cdot d_{\mathrm{p} 2}}{d_{\mathrm{p} 1}}$

In other words, $L$ and $d_{\mathrm{p}}$ are directly proportional. The flow permeability $K_{\mathrm{F}}$ of an LC column, packed with particles, is defined by the Kozeny-Carman equation $[11,12]$ :

$K_{\mathrm{F}}=\frac{4 \cdot F \cdot \eta \cdot L}{d_{\mathrm{c}}^{2} \cdot \pi \cdot \Delta p}=\frac{d_{\mathrm{p}}^{2}}{1,000}$

where $\eta$ is the viscosity of the eluent. $K_{\mathrm{F}}$ is identical in the old and new system if 
the packing qualities are identical. If the same eluent is used the pressure ratio is defined by:

$\frac{\Delta p_{2}}{\Delta p_{1}}=\frac{d_{\mathrm{p} 1}^{2} \cdot L_{2} \cdot F_{2}}{d_{\mathrm{p} 2}^{2} \cdot L_{1} \cdot F_{1}}$

The combination of Eqs. 3 and 6 shows that $L_{2} \cdot u_{2}=L_{1} \cdot u_{1}$, therefore:

$d_{\mathrm{p} 2}=d_{\mathrm{p} 1} \sqrt{\frac{\Delta p_{1}}{\Delta p_{2}}}$

Similarly:

$L_{2}=L_{1} \sqrt{\frac{\Delta p_{1}}{\Delta p_{2}}}$

The breakthrough time $t_{0}$ (and the retention times) of the new column will be decreased. With $L_{2} \cdot u_{2}=L_{1} \cdot u_{1}$, $u=L / t_{0}$ and Eq. 10 we get:

$t_{02}=t_{01} \frac{\Delta p_{1}}{\Delta p_{2}}$

This is exactly the same relationship of breakthrough (and analysis) time as with the non-clever approach; e.g. doubling the pressure will result in halving the time.

Finally, the necessary volume of mobile phase $V_{\text {tot }}$ for the analysis will also decrease, as it is proportional to the column length. If the diameter of the new column will be identical we get, in analogy to Eq. 10:

$V_{\mathrm{tot} 2}=V_{\mathrm{tot} 1} \sqrt{\frac{\Delta p_{1}}{\Delta p_{2}}}$

Exactly the same relationship is valid for the volumes of the individual peaks $V_{\mathrm{P}}$ :

$V_{\mathrm{P} 2}=V_{\mathrm{P} 1} \sqrt{\frac{\Delta p_{1}}{\Delta p_{2}}}$

Eq. 13 follows from the fact that the theoretical plate numbers of both columns are (or should be) identical from the fundamental equation of the plate number:

$N=16 \frac{t_{r}^{2}}{w^{2}}$

with $w$ being the peak width and from Eqs. 4, 9 and 11. (Note again that Eqs. 9-13 are only valid if the reduced

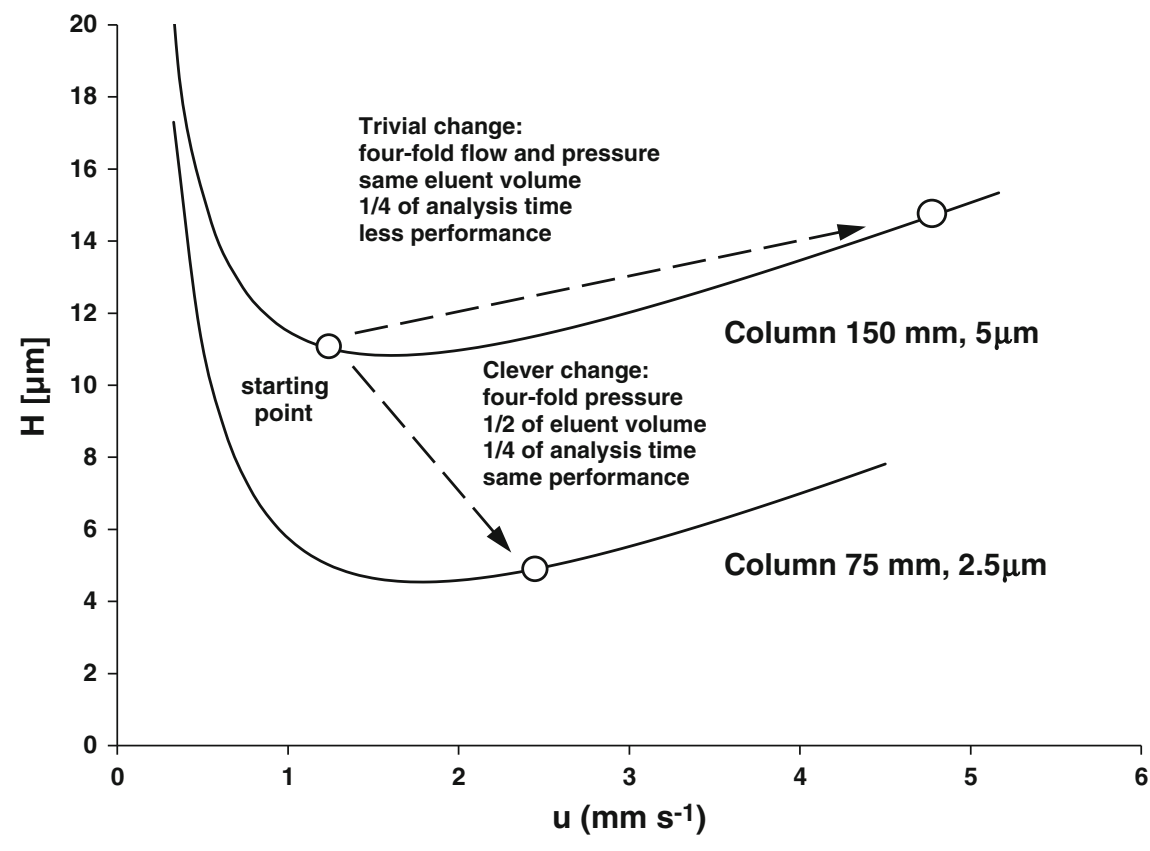

Fig. 1. Trivial and clever possibilities to work at higher pressure, here shown for the case of a fourfold pressure increase, with experimental van Deemter curves of two columns used in this paper. The starting and end points of the "clever change" arrow are experimental values whereas the end point of the "trivial change" arrow is calculated

flow rates and the theoretical plate numbers of both columns are identical.) Working with the new, "clever" column means that the peak volumes will decrease, therefore the extra-column volumes and the detector settings (sampling rate, time constant) should be checked and adapted if necessary.

It was already mentioned by Chen and Kord that UHPLC separations with sub-2 $\mu \mathrm{m}$ particles need less eluent than more conventional separations [13], however, without presenting the theory of the pressure relationships. Fountain et al. [14] show three chromatograms of a drug and its impurities, analysed on three different columns; they come to the same experimental results as shown here but the background is not presented in detail. The "clever pressure" idea was briefly mentioned in a former paper [15] but without a thorough mathematical description.

The set of Eqs. 9-12 allows calculating the parameters of the new separation system if we decide to increase the pressure from $\Delta p_{1}$ to $\Delta p_{2}$. This approach is represented by the "clever change" arrow in Fig. 1. It is advised not to use the maximum possible pressure of the pump or LC system as the value of $\Delta p_{2}$ when planning such a change because a new column may have a worse (or better) permeability than expected; its packing quality, particle size distribution, or flow resistance of fittings and frits cannot be predicted from scratch.

(Note: the above-mentioned Halász diagrams [9] allow finding the new conditions of column length, particle diameter and breakthrough time at a glance when the theoretical plate number is to be kept constant while another pressure will be applied. They are drawn for the van Deemter optimum but their information approach is also valid for other flow velocities.)

In this paper, the "clever pressure" proposal is illustrated by using three different columns with $5,3.5$ and $2.5 \mu \mathrm{m}$ packing and appropriate lengths to give constant numbers of theoretical plates. The experimental and predicted data are shown in Table 1. Two different analytical problems were studied on all three columns, namely the separation of seven doping agents and the separation of six drugs. 
Table 1. Data of the columns used in this study expected to give the same separation performance

\begin{tabular}{|llll|}
\hline Parameter & Original column & New column 3.5 & New column 2.5 \\
\hline Particle diameter & $5 \mu \mathrm{m}$ & $3.5 \mu \mathrm{m}$ & $2.5 \mu \mathrm{m}$ \\
Column length & $150 \mathrm{~mm}$ & $100 \mathrm{~mm}$ & $75 \mathrm{~mm}$ \\
Relative flow rate & 1.0 & 1.4 & 2.0 \\
Relative pressure & 1.0 & 2.0 & 4.0 \\
Relative analysis time & 1.0 & 0.5 & 0.25 \\
Relative eluent volume & 1.0 & 0.7 & 0.5 \\
\hline
\end{tabular}

Particle diameters and column lengths are technical data, the other numbers are calculated from theory

\section{Experimental}

\section{Chemicals and Reagents}

The seven doping agents used in this work were generously supplied by the "Swiss Laboratory for Doping Analyses" (Epalinges, Switzerland): metoprolol, esmolol, dexamethasone, indapamide, piretanide, probenecid, and bendroflumethiazide. Stock solutions of each analyte of $1 \mathrm{mg} \mathrm{mL}^{-1}$ in methanol were prepared and appropriately diluted with pure water to attain final concentrations in the mixture of $2 \mu \mathrm{g} \mathrm{mL}^{-1}$ for dexamethasone, indapamide, piretanide, probenecid, $5 \mu \mathrm{g} \mathrm{mL}^{-1}$ for bendroflumethiazide, and $50 \mu \mathrm{g} \mathrm{mL}^{-1}$ for metoprolol and esmolol.

The six pharmaceutical compounds were from Sigma-Aldrich (Steinheim, Germany): flunitrazepam, prilocaine, tetracaine, donepezil, bupropion, and bupivacaine. Again, the stock solutions of each analyte with $1 \mathrm{mg} \mathrm{mL}^{-1}$ in methanol were prepared and appropriately diluted with pure water to attain final concentrations in the mixture of $1 \mu \mathrm{g} \mathrm{mL}{ }^{-1}$ for flunitrazepam, $2 \mu \mathrm{g} \mathrm{mL}^{-1}$ for prilocaine and bupropion, $5 \mu \mathrm{g} \mathrm{mL}^{-1}$ for tetracaine, and $10 \mu \mathrm{g} \mathrm{mL}^{-1}$ for donepezil and bupivacaine.

Acetonitrile was of LC grade from Panreac Quimica (Barcelona, Spain). Ammonium hydroxide and acetic acid were provided by Sigma-Aldrich (Buchs, Switzerland). Water was from a Milli-Q Water Purification System (Millipore Bedford, MA, USA).

Acetate buffer $50 \mathrm{mM}$ was prepared with the necessary volume of acetic acid and $\mathrm{pH}$ adjustment to 4.0 with ammonium hydroxide. Ammonia buffer
$50 \mathrm{mM}$ was prepared with the necessary volume of ammonium hydroxide and $\mathrm{pH}$ adjustment to 9.0 with acetic acid. In this work, the $\mathrm{pH}$ was measured with a Metrohm pH meter (Herisau, Switzerland) and each prepared buffer had a buffer capacity higher than $5 \mathrm{mM}$ per $\mathrm{pH}$ unit.

\section{Instrumentation}

Separations were performed with a Merck LaChrom system (Merck, Darmstadt, Germany) consisting of two L-7100 programmable pumps, an L-7200 autosampler with a $100 \mu \mathrm{L}$ loop (injection between 10 and $20 \mu \mathrm{L}$ in the partial loop mode), an L-7614 on-line degasser, an L-7400 UV-VIS programmable detector set to a wavelength of 254 or $230 \mathrm{~nm}$ for doping agents and pharmaceutical compounds, respectively, and a Jetstream 2 plus column oven. The UV-VIS detector contained a $14 \mu \mathrm{L}$ standard flow cell, the time constant and data sampling rate were set to the lowest (i.e. $0.1 \mathrm{~s}$ ) and highest values (i.e. $20 \mathrm{~Hz}$ ), respectively. Data acquisition, data handling and instrument control were performed with the D-7000 HPLC System Manager Software. The extra-column volume $\left(V_{\text {ext }}\right)$ of this LC configuration was experimentally estimated using a zero-dead volume connector and was determined to be $115 \mu \mathrm{L}$.

\section{Columns}

The columns used throughout this study have similar chemistries, namely Waters
Xbridge $\mathrm{C}_{18}$. Various dimensions of column length and particle diameter were selected, namely, $4.6 \mathrm{~mm}$ i.d. $\times 150 \mathrm{~mm}$, $5 \mu \mathrm{m} ; 4.6 \mathrm{~mm}$ i.d. $\times 100 \mathrm{~mm}, 3.5 \mu \mathrm{m}$; and $4.6 \mathrm{~mm}$ i.d. $\times 75 \mathrm{~mm}, 2.5 \mu \mathrm{m}$, all from Waters (Milford, MA, USA). In all three cases, the quotients of column length and particle diameter give approx. $3 \times 10^{4}$; therefore their number of theoretical plates should be identical if the packing quality is identical. (Note that the inner diameter of the columns was identical.)

\section{Separation Conditions}

Two different mobile phases were used, both of them in isocratic mode. The doping agents were separated with an eluent of $30 \%$ acetonitrile $/ 70 \%$ acetate buffer $50 \mathrm{mM} \mathrm{pH} \mathrm{4.} \mathrm{The} \mathrm{drugs} \mathrm{were}$ separated with $50 \%$ acetonitrile $/ 50 \%$ ammonia buffer $50 \mathrm{mM} \mathrm{pH} \mathrm{9.} \mathrm{Separa-}$ tion temperature was $30^{\circ} \mathrm{C}$ in all cases. The flow rates were set according to Eq. 4, i.e. as noted in Table 1: the first column with $5 \mu \mathrm{m}$ packing was used at $0.7 \mathrm{~mL} \mathrm{~min}^{-1}$, the second one with $3.5 \mu \mathrm{m}$ at $1.0 \mathrm{~mL} \mathrm{~min}^{-1}$, and the third one with $2.5 \mu \mathrm{m}$ at $1.4 \mathrm{~mL} \mathrm{~min}^{-1}$.

\section{Determination of van Deemter Curves}

The experimental van Deemter curves presented in Fig. 1 were determined in order to compare the chromatographic performance of columns that differ in length and particle size. These curves were constructed by measuring the chromatographic efficiency, $N$, of a model neutral compound, butylparaben, on the different columns at various mobile phase flow rates ranging

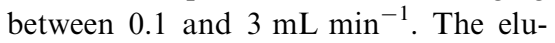
ent was $40 \%$ acetonitrile $/ 60 \%$ water. The breakthrough time $t_{0}$, needed for the calculation of the linear flow rate, was determined with uracil. The experimental curves were fitted with the Solver function of Excel (Microsoft 2003) using least square regression based on the equation $H=A+B /$ $u+C u$. 


\section{Results and Discussion}

\section{van Deemter Curves}

Two of the $H / u$ curves for butylparabene are shown in Fig. 1, namely those for the 5 and $2.5 \mu \mathrm{m}$ phases. They have minimum theoretical plate heights $H$ of 11 and $5 \mu \mathrm{m}$, respectively, or reduced plate heights $h$ of 2.2 and $2.0\left(h=H / d_{\mathrm{p}}\right)$. In comparison, the van Deemter curve of the $3.5 \mu \mathrm{m}$ phase, not shown in Fig. 1, was too high with a minimum at $9.5 \mu \mathrm{m}$ (or $h=2.7$ ), i.e. the chromatographic performance of this column is relatively worse compared to the other two.

\section{Pressure Relations}

As predicted by Table 1 , the $3.5 \mu \mathrm{m}$ column is expected to run at twice the pressure of the $5 \mu \mathrm{m}$ one, and the $2.5 \mu \mathrm{m}$ column should give four times the pressure. The first column with $5 \mu \mathrm{m}$ pack-

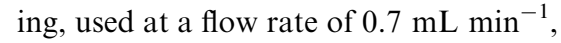
gave a pressure drop of 74 and of 67 bar, respectively, for the two different eluents (doping agents vs. drugs). The second column with $3.5 \mu \mathrm{m}$ was used at $1.0 \mathrm{~mL} \mathrm{~min}^{-1}$. The resulting pressures were 161 and 149 bar, respectively, which is slightly higher than what was calculated using Eq. 8; the calculation predicts 148 and 134 bar. The column with $2.5 \mu \mathrm{m}$ packing was used at $1.4 \mathrm{~mL} \mathrm{~min}^{-1}$, giving pressures of 297 and 261 bar, respectively, values which match the predictions given by Eq. 8 .

The total porosities of the columns are practically identical, namely 0.60 , 0.58 , and 0.59 (calculated from the respective volume flow rate, the breakthrough time as determined with uracil, the column diameter, and the column length). Thus, the linear velocities $u$ are proportional to the volume flow rates $F$. From the pressure data given above it follows that the $3.5 \mu \mathrm{m}$ column has a lower permeability than the other two. Therefore, its experimental performance, with regard to the predicted pressure, is poorer than expected from theory. The $2.5 \mu \mathrm{m}$ column, on the other hand, fits perfectly into the data of Table 1 .

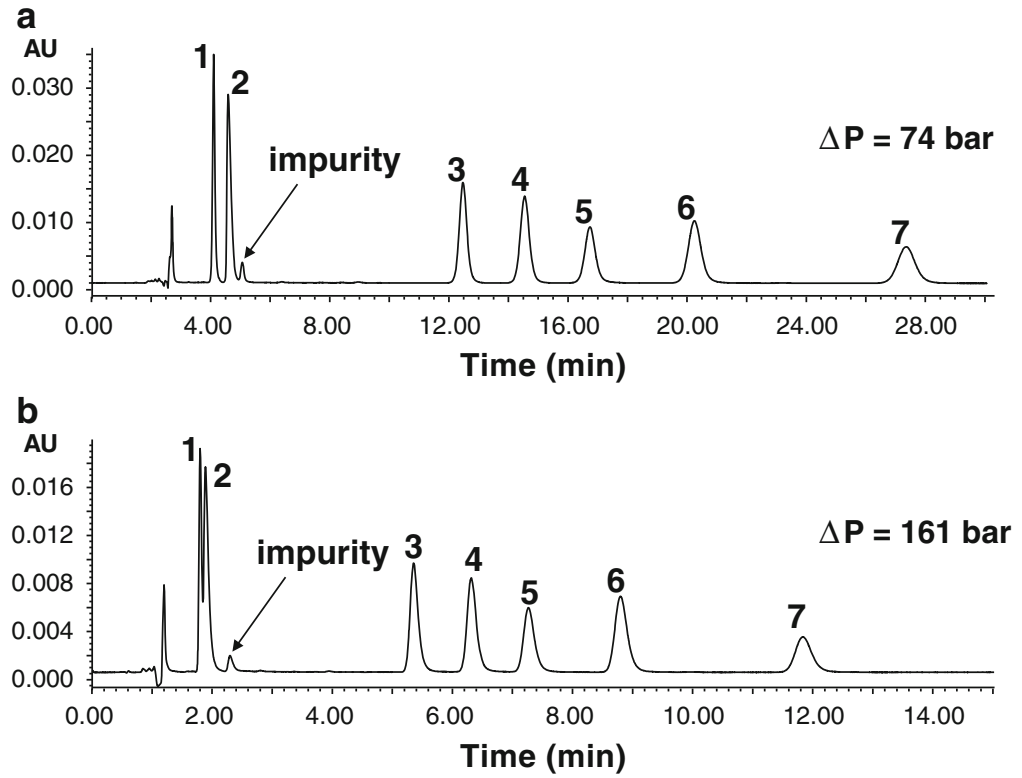

$5 \mu \mathrm{m}$

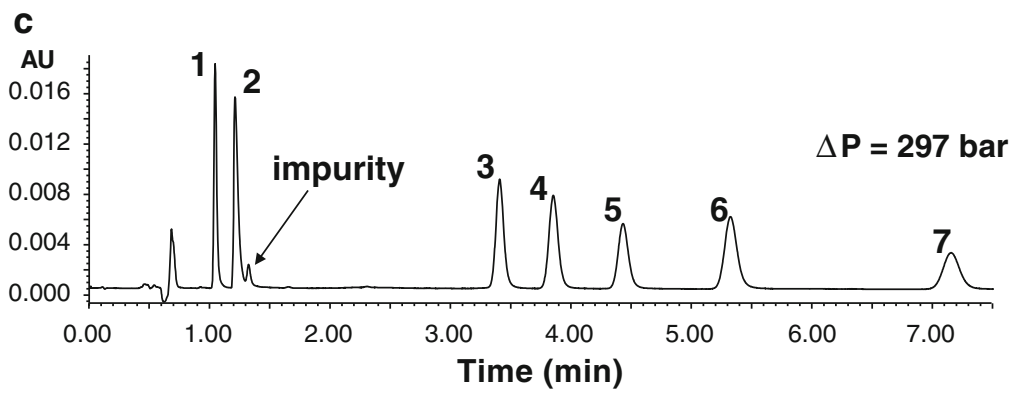

$2.5 \mu \mathrm{m}$

Fig. 2. Chromatograms obtained for the mixture of seven doping agents with various Xbridge C18 columns, using an isocratic mobile phase consisting of $30 \%$ acetonitrile $/ 70 \%$ acetate buffer $50 \mathrm{mM} \mathrm{pH} \mathrm{4}$, a temperature of $30^{\circ} \mathrm{C}$, and UV detection at $254 \mathrm{~nm}$. a Column of $4.6 \mathrm{~mm}$ i.d. $\times 150 \mathrm{~mm}, 5 \mu \mathrm{m}$, flow of $0.7 \mathrm{~mL} \mathrm{~min}{ }^{-1}$ and injection volume of $20 \mu \mathrm{L}$. b Column of $4.6 \mathrm{~mm}$ i.d. $\times 100 \mathrm{~mm}, 3.5 \mu \mathrm{m}$, flow of $1.0 \mathrm{~mL} \mathrm{~min}^{-1}$ and injection volume of $13 \mu \mathrm{L}$. c Column of $4.6 \mathrm{~mm}$ i.d. $\times 75 \mathrm{~mm}, 2.5 \mu \mathrm{m}$, flow of $1.4 \mathrm{~mL} \mathrm{~min}^{-1}$ and injection volume of $10 \mu \mathrm{L}$. Peaks: $\mathbf{1}=$ metoprolol, $\mathbf{2}=$ esmolol, $\mathbf{3}=$ dexamethasone, $\mathbf{4}=$ indapamide, $\mathbf{5}=$ probenecid, $\mathbf{6}=$ piretanide, $7=$ bendroflumethiazide

\section{Separation Performances}

Figure 2 compares the separations of the doping agents with the three columns. The chromatograms end at different times, decreasing from top to bottom, but at identical retention factors, namely $k=14$. (The breakthrough times are approx. 2, 1, and $0.5 \mathrm{~min}$, the analysis times are 30,15 , and $7.5 \mathrm{~min}$.) The chromatograms are similar, as expected, but small differences are present. The $3.5 \mu \mathrm{m}$ phase is less retentive than the $5 \mu \mathrm{m}$ phase, therefore compounds 1 and 2 are not baseline resolved with the eluent in use. The $2.5 \mu \mathrm{m}$ phase is slightly more retentive.
Figure 3 compares the separations of the drugs. The separations end at $k=9$. Again, the $3.5 \mu \mathrm{m}$ phase is less retentive than the $5 \mu \mathrm{m}$ one whereas the $2.5 \mu \mathrm{m}$ phase is slightly more retentive. In fact, the $3.5 \mu \mathrm{m}$ phase is poorer than the other two. Its theoretical plate number is lower (or its reduced plate height in the van Deemter optimum is higher, as mentioned above) compared to the others. In addition, its selectivity for donezepil and bupropion (peaks $\mathbf{4}$ and $\mathbf{5}$ in Fig. 3) is comparatively low: the separation factor $\alpha_{4,5}$ is 1.07 whereas it is 1.12 and 1.17 for the 5 and $2.5 \mu \mathrm{m}$ columns, respectively. The combined effects of lower theoretical plate number, lower 


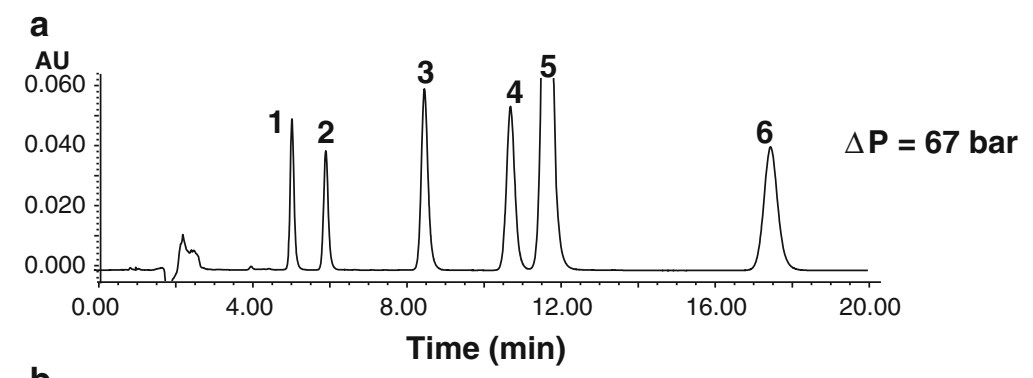

retentivity but at the cost of poorer resolution.

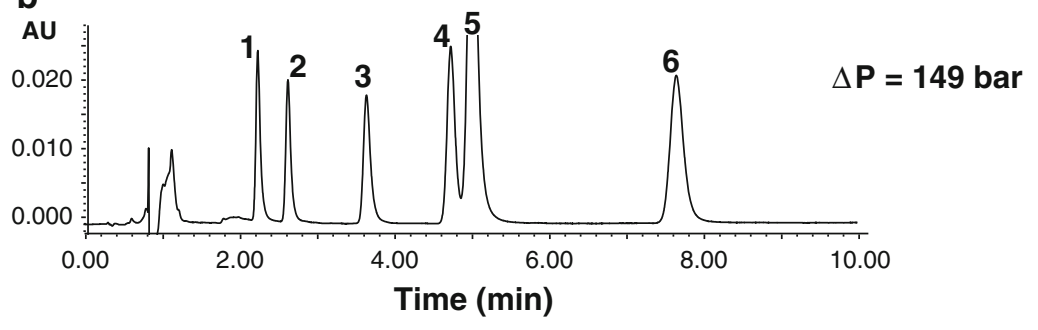

$3.5 \mu \mathrm{m}$

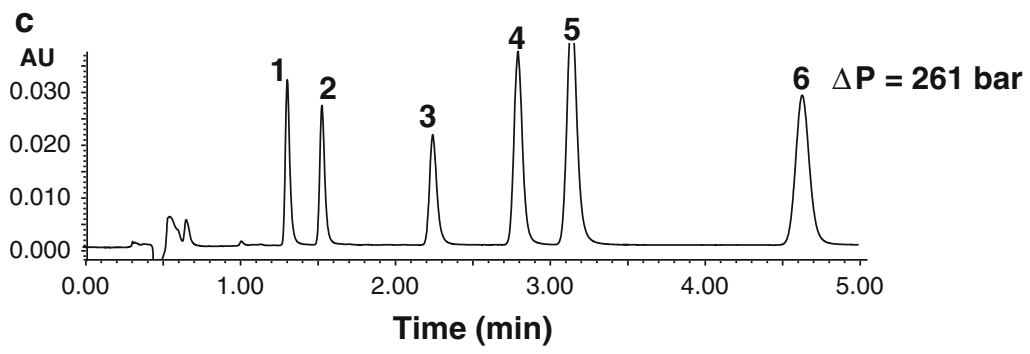

$2.5 \mu \mathrm{m}$

Fig. 3. Chromatograms obtained for the mixture of six pharmaceutical compounds with various Xbridge C18 columns, using an isocratic mobile phase consisting of $50 \%$ acetonitrile $/ 50 \%$ ammonia buffer $50 \mathrm{mM} \mathrm{pH} \mathrm{9,} \mathrm{a} \mathrm{temperature} \mathrm{of} 30^{\circ} \mathrm{C}$, and UV detection at $230 \mathrm{~nm}$. a Column of $4.6 \mathrm{~mm}$ i.d. $\times 150 \mathrm{~mm}, 5 \mu \mathrm{m}$, flow of $0.7 \mathrm{~mL} \mathrm{~min}^{-1}$ and injection volume of $20 \mu \mathrm{L}$. b Column of $4.6 \mathrm{~mm}$ i.d. $\times 100 \mathrm{~mm}, 3.5 \mu \mathrm{m}$, flow of $1.0 \mathrm{~mL} \mathrm{~min}{ }^{-1}$ and injection volume of 13 $\mu$ L. c Column of $4.6 \mathrm{~mm}$ i.d. $\times 75 \mathrm{~mm}, 2.5 \mu \mathrm{m}$, flow of $1.4 \mathrm{~mL} \mathrm{~min}{ }^{-1}$ and injection volume of $10 \mu \mathrm{L}$. Peaks: $\mathbf{1}=$ flunitrazepam, $\mathbf{2}=$ prilocaine, $\mathbf{3}=$ tetracaine, $\mathbf{4}=$ donepezil, $\mathbf{5}=$ bupropion, $\mathbf{6}=$ bupivacaine Meyer, The clever use of pressure in column liquid chromatography

retentivity and lower selectivity lead to the incomplete resolution of peaks 4 and 5 on the $3.5 \mu \mathrm{m}$ column (Fig. $3 \mathrm{~b}$ ). In addition, this column shows a tendency towards peak tailing. However, the overall theoretical expectations are also obtained in this case.

The $5 / 2.5 \mu \mathrm{m}$ column pair is a perfect example to demonstrate the validity of the "clever pressure theory". The chromatograms look almost identical and the pressure ratios agree with the theoretical predictions, with a four-fold increase when using the $2.5 \mu \mathrm{m}$ packing.

\section{Eluent Volumes and Analysis Times}

Column (a) with the $5 \mu \mathrm{m}$ packing needs $20 \mathrm{~mL}$ of eluent volume $(28.5 \mathrm{~min} \times$
$0.7 \mathrm{~mL} \mathrm{~min}^{-1}$ ) for the separation of the doping agents and $13 \mathrm{~mL}$ for the drugs. As discussed above, the chromatograms obtained with column (b) cannot be compared directly; less eluent than predicted is needed but the resolutions are poorer. Column (c) with the $2.5 \mu \mathrm{m}$ packing needs slightly more eluent than predicted, namely 10.4 and $6.7 \mathrm{~mL}$ instead of 10.0 and $6.5 \mathrm{~mL}$, respectively. These deviations from theory are negligible.

The total analysis time of the $2.5 \mu \mathrm{m}$ column agreed with the predictions, again with the small deviation just discussed. $1 / 4$ of $28.5 \mathrm{~min}$ is $7.1 \mathrm{~min}$ (doping agents), when the predicted value was $7.4 \mathrm{~min}$. $1 / 4$ of $18 \mathrm{~min}$ is $4.5 \mathrm{~min}$ (drugs) but $4.8 \mathrm{~min}$ was predicted. The total analysis time of the $3.5 \mu \mathrm{m}$ column was lower than expected due to its lower

\section{Conclusions}

The experimental data demonstrates that the "clever pressure approach" works in practice. It results in shorter analysis times and less solvent consumption without deteriorating the separation if a new column with identical quality (defined as reduced theoretical plate height as well as retentivity and permeability) is available. The new column dimensions, i.e. its stationary phase diameter and length, are calculated easily with the simple pressure relationships show in Eqs. 9 and 10. It may be necessary to adapt the extra-column volumes of the instrument and the detector settings to the new conditions since the peaks will be narrower with the new column.

\section{Acknowledgment}

The chromatograms and the van Deemter curves were kindly generated by Kevin P. Sullivan, Davy Guillarme and Jean-Luc Veuthey from the School of Pharmaceutical Sciences, University of Geneva, University of Lausanne, Switzerland.

\section{References}

1. Mazzeo JR, Neue UD, Kele M, Plumb RS (2005) Anal Chem 77:460A-467A. doi:10.1021/ac053516f

2. Nguyen D, Guillarme D, Rudaz S, Veuthey JL (2006) J Sep Sci 29:18361848. doi:10.1002/jssc. 200600189

3. Van Deemter JJ, Zuiderweg FJ, Klinkenberg A (1956) Chem Eng Sci 5:271-289. doi:10.1016/0009-2509(56)80003-1

4. Bristow PA, Knox JH (1977) Chromatographia 10:279-289. doi:10.1007/ BF02263001

5. Wilke CR, Chang P (1955) Am Inst Chem Engr J 1:264-270

6. Martin M, Eon C, Guiochon G (1974) J Chromatogr 99:357-376. doi:10.1016/ S0021-9673(00)90870-7

7. Martin M, Eon C, Guiochon G (1975) J Chromatogr 108:229-241. doi:10.1016/ S0021-9673(00)84666-X

8. Martin M, Eon C, Guiochon G (1975) J Chromatogr 110:213-232. doi: 10.1002/ange. 19820940105 
9. Halász I, Görlitz G (1982) Angew Chem Int Ed 21:50-61. doi:10.1002/anie.198200501

10. Meyer VR (2010) Practical high-performance liquid chromatography, 5th edn. Wiley, Chichester, pp 54-55

11. Kozeny J (1927) Sitzungsber Akad Wiss Wien 136:271-306
12. Carman PC (1937) Trans Inst Chem Eng Lond 15:150-166

13. Chen S, Kord A (2009) J Chromatogr A 1216:6204-6209. doi:10.1016/j.chroma. 2009.06.084

14. Fountain KJ, Neue UD, Grumbach ES, Diehl DM (2009) J Chromatogr A
1216:5979-5988. doi:10.1016/j.chroma. 2009.06.044

15. Guillarme D, Veuthey JL, Meyer VR (2008) LC GC Eur 21:322-327 